\title{
Author Index to Volume 9
}

Agid, Y: 171 (May)

Allard, P: 105, 113, 119 (May)

Andermann, E: 131, 189, 195 (May)

Bachevalier, J: 37 (Feb)

Ball, M.J.: 303 (Aug)

Barabe, L: 155 (May)

Barbeau, A: 53 (Feb), 95, 141, 151, 175, 179,

$181,189,191,195,205,209,217,231,235$,

239, 243 (May)

Barbeau, C.M.: 175 (May)

Benoit, B.G.: 321 (Aug), 409 (Nov)

Bertrand, M.J.: 231, 239 (May)

Bilbao, J.M.: 49 (Feb)

Blache, D: 181, 191 (May)

Blair, J.D.: 31 (Feb)

Boileau, J: 41 (Feb)

Botez, M.I.: 37 (Feb)

Bouchard, J.P.: 127, 217, 231, 239 (May)

Bouchard, R: 127, 217, 231, 239 (May)

Bouthillier, D: 191 (May)

Brown, W.F.: 391 (Nov)

Campanella, G: 147 (May)

Carlen, P.L.: 89 (May)

Carpenter, B: 435 (Nov)

Charbonneau, M: 209, 235 (May)

Choo, S.H.: 321 (Aug)

Chouza, C: 141 (May)

Cloutier, T: 209, 217, 235 (May)

Cochrane, D.D.: 409 (Nov)

Codina, M: 99 (May)

Coffey, C.E.: 45 (Feb)

Coupland, S.G.: 325, 331 (Aug)

Cousineau, J: 165 (May)

Daigle, M: 421 (Nov)

Dallaire, L: 15I, 155, 165 (May)

Daly, L.L.: 439 (Nov)

Dansereau, J: 105 (May)

Davignon, J: 181, 191 (May)

De Champlain, J: 195 (May)

de Mello, D.E.: 31 (Feb)

De Michele, G: 175, 179 (May)

Desmet, Y: 171 (May)

DiPaolo, T: 421 (Nov)

Dobrescu, O: 345 (Aug)

Dolan, R.A.: 61 (Feb)

Dolman, C.L.: 439 (Nov)

Duhaime, M: 105, 113, 119 (May)

Duplantis, F: 41 (Feb)

Dupont, A: 421 (Nov)

Durity, F: 409 (Nov)

Dykes, R.W.: 9 (Feb)
Eisen, A: 65 (May)

Ferguson, G.G.: 409 (Nov)

Fewer, D: 409 (Nov)

Filla, A: 147 (May)

Findley, L.J.: 99 (May)

Fletcher, J.P.: 435 (Nov)

Fontaine, G: 155, 165 (May)

Fornazzari, L: 89 (May)

Garaizar, C: 401 (Nov)

Gauthier, G.L.: 231, 239 (May)

Geoffroy, G: 105, 113, 119, 151, 155, 165, 195 (May)

Gordon, A.S.: 49 (Feb)

Gordon, P.A.: 27 (Feb)

Grignon, B: 165 (May)

Hardy, J: 375 (Nov)

Harvey, C: 189 (May)

Hashimoto, S.A.: 21 (Feb)

Huang, Y.S.: 181 (May)

Hugenholtz, H: 321 (Aug)

Hull, K.L.: 85 (May)

Humphreys, P: 435 (Nov)

Hunter, K.M.: 409 (Nov)

Jolicoeur, F.B.: 175, 179 (May)

Joyal, C: 37 (Feb)

Kandel, R.A.: 49 (Feb)

Keene, D.L.: 307 (Aug), 435 (Nov)

Khan, M.I.: 409 (Nov)

Kirkham, T.H.: 325, 331 (Aug)

Krol, R: 195 (May)

Lachapelle, J: 41 (Feb)

Lamarche, J: 137 (May)

Lane, R.J.M.: 85 (May)

Lang, A.E.: 313 (Aug)

Lapous, D: 221 (May)

Larbrisseau, A: 345 (Aug)

La Torre, P: 99 (May)

Launay, M: 221 (May)

Laverdière, M: 41 (Feb)

Lee, R.G.: 1, 3 (Feb)

Lemieux, B: 137 (May)

Lhermitte, F: 171 (May)

Linschoten, L: 429 (Nov)

Lopez-Hernandez, A: 401 (Nov)

Loriette, C: 221 (May)

Luneau, C: 137 (May)

\author{
Maag, U: 37 (Feb) \\ Marois, P: 151 (May) \\ Marsden, C.D.: 313 (Aug) \\ Martin, W.R.W.: 21 (Feb) \\ Martinez-Lage, J.M.: 99 (May) \\ Massey, W.W.: 45 (Feb) \\ McDonald, W.I.: 381 (Nov) \\ McMenamin, J.B.: 131 (May) \\ McPherson, T.A.: 27 (Feb) \\ Mear, J.Y.: 171 (May) \\ Mehta, A: 189 (May) \\ Melancon, S.B.: 151, 155, 165, 195 (May) \\ Merkel, A.D.: 205 (May) \\ Messer, A: 185 (May) \\ Metrakos, K: 307 (Aug) \\ Mohr, G: 409 (Nov) \\ Monckton, G: 79 (May) \\ Moser, H.W.: 449 (Nov) \\ Murray, T.J.: 453 (Nov) \\ Nandon, G: 209 (May) \\ Norman, M.G.: 339 (Aug), 443 (Nov)
}

Olanow, C.W.: 45 (Feb), 85 (May)

Olivenstein, R: 195 (May)

O'Neill, B.P.: 449 (Nov)

Pasternac, A : 195 (May)

Patenaude, F: 209 (May)

Pehowich, E: 79 (May)

Peticlerc, R: 195 (May)

Potier, M: 151, 155, 165 (May)

Pourcher, E: 53 (Feb)

Pritzker, K.P.H.: 49 (Feb)

Proulx, A: 155 (May)

Raso, J.V.: 105 (May)

Raulin, J: 221 (May)

Richard, M.T.: 321 (Aug)

Rosenblatt, B: 429 (Nov)

Roses, A.D.: 85 (May)

Ross, D.R.: 45 (Feb)

Rousseau, S: 41 (Feb)

Roy, A: 141 (May)

Roy, L.E.: 41 (Feb)

Russel, N.A.: 321 (Aug) 
Saint-Martin, M: 41 (Feb)

Salisachs, P: 99 (May)

Sarnat, H.B.: 31 (Feb)

Schechter, P.J.: 171 (May)

Sheehy, M.P.: 313 (Aug)

Sherwin, A: 307 (Aug)

Siddiqui, S.Y.: 31 (Feb)

Sirois, J.P.: 113 (May)
Taylor, M.J.: 131 (May), 429 (Nov)

Tell, G: 171 (May)

Thiry, P.S.: 105, 113, 119 (May)

Tolis, G: 189 (May)

Vanasse, M: 151, 155, 165 (May)

Ventureyra, E.C.G.: 321 (Aug)

Vezina, J.G.: 127 (May)
Wagniart, P.L.: 195 (May)

Warren, S.A.: 415 (Nov)

Warren, K.G.: 27 (Feb), 415 (Nov)

Watters, G.V.: 131 (May), 307 (Aug)

Watts, A.R.: 409 (Nov)

Weir, B.K.A.: 409 (Nov)

Wheelock, W.B.: 409 (Nov)

Wong, P: 181 (May)

Yates, S.K.: 391 (Nov) 